\title{
ARTICLE OPEN \\ Kinetics of thermally activated triplet fusion as a function of polymer chain packing in boosting the efficiency of organic light emitting diodes
}

\author{
Amrita Dey ${ }^{1}$, Naresh Chandrasekaran ${ }^{1,2,3}$, Dwaipayan Chakraborty ${ }^{4}$, Priya Johari ${ }^{4}$, Christopher R. McNeill ${ }^{3}$, Akshay Rao (iD ${ }^{5}$ and \\ Dinesh Kabra (iD ${ }^{1}$
}

Understanding the photophysical process governing the operation of the organic light emitting diodes (OLEDs) and how they are affected by film morphology is crucial to the efficient design of future OLEDs. In particular, delayed fluorescence (DF), is known to contribute a significant fraction of the light emission from polymer-based OLEDs, but its mechanism remains unclear. Here, we investigate the origin of DF in the state of the art OLED polymer Poly (9, 9-dioctylfluorene-alt-benzothiadiazole) (F8BT), under both optical and electrical excitation using time-resolved emission spectroscopy (TRES) as a function of film thickness, excitation fluence, magnetic-field, and temperature. The temperature dependence of the DF for various film thicknesses suggests that thermally activated triplet migration is the dominant process controlling DF at room temperature. We found that thermal activation energy $\left(E_{\text {eff }}\right)$ of triplet migration decreases from $179 \pm 31 \mathrm{meV}$ to $86 \pm 11 \mathrm{meV}$ as film thickness varied from $\sim 110 \mathrm{~nm}$ to $\sim 560 \mathrm{~nm}$, respectively. The $E_{\text {eff }}$ of triplet migration is found to be a function of the molecular packing of polymer chains as determined from synchrotron grazing incidence wide angle x-ray scattering (GIWAXS) studies and steady-state photoluminescence studies. Quantum chemical calculations of reorganization energy and singlet-triplet exchange energy gap in F8BT molecule as a function of the dihedral angle between donor \& acceptor moiety also confirm the experimental results. Our results show that DF in polymer OLEDs is significantly affected by parameters such as the film thickness and disorder, allowing for a high degree of control over the underlying photophysics to be achieved.

npj Flexible Electronics (2018)2:28; doi:10.1038/s41528-018-0042-0

\section{INTRODUCTION}

Since the discovery of Organic light emitting diodes (OLEDs) by Tang et al. ${ }^{1}$ in 1989 followed by the electroluminescence in conjugate polymer-based LED by Burroughs et al. ${ }^{2}$ in 1990, these devices have now been successfully used in consumer display devices. However, the demands of increased efficiency with color purity and device stability are still high for displays. There is also the beginning of new era for solid-state lighting using OLEDs. Mainly four parameters control the efficiency of OLEDs. They are charge carrier injection efficiency $(\gamma)$, singlet generation yield $\left(\eta_{S / T}\right)$, photoluminescence efficiency of the emissive layer $\left(\eta_{P L}\right)$, and the outcoupling factor $\left(\eta_{\text {out }}\right)\left(E Q E=\gamma \times \eta_{P L} \times \eta_{S / T} \times \eta_{\text {out }}\right) \cdot{ }^{3}$ Light emission properties of fluorescent small molecular semiconductor or conjugated polymer are intrinsically restricted to $25 \%$ singlet generation yield $\left(\eta_{S / T}\right)$ under spin-independent electrical charge injection following the spin statistical 1:3 singlet: triplet exciton formation ratio. Though in phosphorescent materials-based OLEDs radiative yield can become $100 \%$, material stability is relatively poor with efficiency roll-off at higher current density. ${ }^{4}$ Hence, fluorescent materials with higher singlet yield are a favorable choice. Singlet excitons produced via triplet-triplet annihilation (TTA) or triplet fusion (TF) method has been shown to enhance the
OLED efficiency beyond statistical spin limit $(\sim 5 \%) .^{5-7}$ However, the total singlet generation yield, in this case, is limited to $\sim 62.5 \% .^{5}$ Another mechanism for getting an extra boost in singlet generation yield is thermally activated delayed fluorescence (TADF) in OLEDs. ${ }^{8,9}$ In TADF molecules there is small singlet-triplet exchange energy, which allows the triplet excitons to back transfer at the singlet manifold via reverse intersystem crossing (RISC) with the help of thermally activated process. However, there are many organic semiconductor systems where TTA and TADF coexist, which require a careful investigation to determine the role of these individual processes. ${ }^{10,11}$ Photophysical investigation of DF to understand the excitonic processes is thus important in such systems. Not only the mechanism of triplet utilization in harvesting singlet excitons in fluorescent OLEDs play an important role, but also temperature-assisted triplet energy transfer to enhance the DF is another critical aspect of controlling device efficiency. Since, triplet migration depends on polymer film morphology and hopping frequency between polymer chains. ${ }^{12-14}$

Here, we investigate the origin of delayed fluorescence in donor-acceptor-based co-polymer system Poly(9,9-dioctylfluorene-alt-benzothiadiazole) (F8BT), known for demonstrating excellent EQE efficiency $\sim 9 \%$ as reported by $\mathrm{us}^{3}$ and as also reported by Lu et al. ${ }^{15}$ This is far beyond the spin statistical EQE limit $~ 3.5 \%$ for

\footnotetext{
'Department of Physics, Indian Institute of Technology Bombay, Powai, Mumbai 400076, India; ${ }^{2}$ IITB-Monash Research Academy, IIT Bombay, Mumbai 400076, India; ${ }^{3}$ Department of Materials Science and Engineering, Monash University, Wellington Road, Clayton, Victoria 3800, Australia; ${ }^{4}$ Department of Physics, School of Natural Sciences, Shiv Nadar University, Greater Noida, Gautam Buddha Nagar, U.P. 201314, India and ${ }^{5}$ Cavendish Laboratory, University of Cambridge, JJ Thomson Avenue, Cambridge CB3 0HE, UK Correspondence: Amrita Dey (amritadey.phy@iitb.ac.in) or Priya Johari (priya.johari@snu.edu.in) or Dinesh Kabra (dkabra@iitb.ac.in)
} 
a
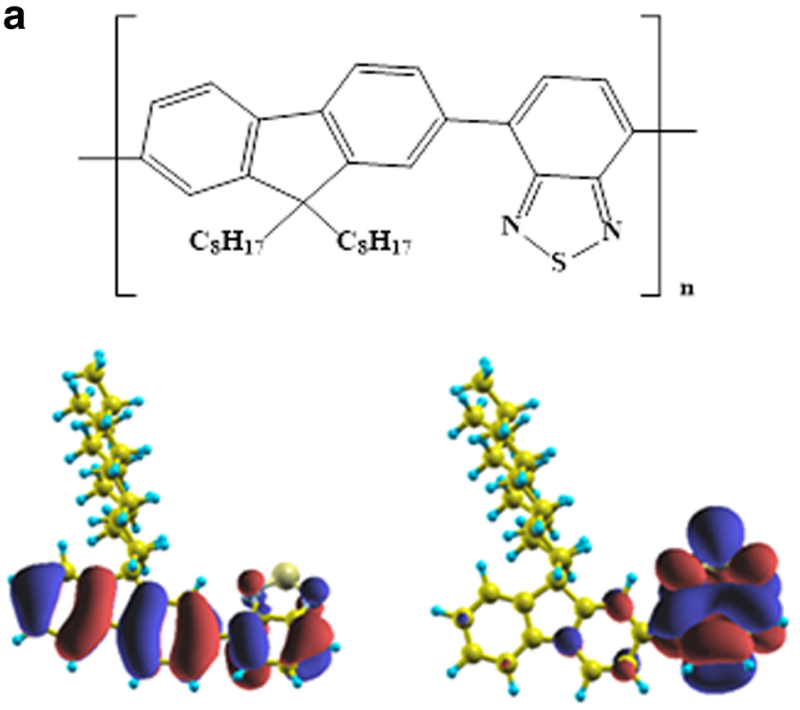

HOMO

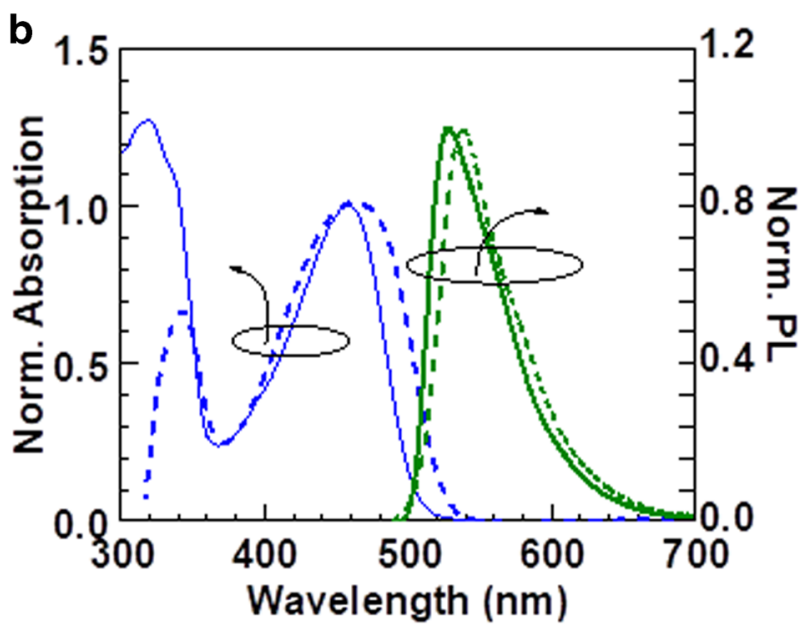

Fig. 1 Chemical structure and steady state characteristics of F8BT. a Typical chemical structure of F8BT co-polymer system and calculated HOMO and LUMO spatial distribution in F8BT, with isosurface value \pm 0.02 a.u. at the TD-B3LYP/6-311 $+\mathrm{G}(\mathrm{d}, \mathrm{p})$ level of theory. $\mathbf{b}$ Absorption spectra of F8BT solution (blue solid line) and solid film (blue dashed line). Photoluminescence (PL) spectra of the F8BT solution in $p$-xylene (green solid line) and in the solid film (green dashed line). The absorption and PL in solid is red-shifted from the solution

$\mathrm{F} 8 \mathrm{BT}^{3,6}$ in the flat OLED architecture without any outcoupling enhancement. Further, with enhanced outcoupling, EQE in F8BT PLED reached up to $18 \%$, as reported by Song et al. ${ }^{16}$ Most interestingly, it was found that these PLEDs perform best with thicknesses of $\sim 1 \mu \mathrm{m}$, which is pretty unusual (one order higher) for PLED research community. ${ }^{6,15,16}$ Therefore, a careful investigation of DF origin under optical and electrical excitation is needed in detail to correlate it with results of high EQE PLEDs for such a thick film. The optical properties of this polymer system was investigated in past, but the studies were mainly restricted in prompt fluorescence (PF) and exciton annihilation processes. ${ }^{17-19}$ The origin of DF in electrically excited F8BT polymer light emitting diodes (PLEDs) has earlier been assigned to the TTA. ${ }^{6}$ However, triplet transfer processes as a function of solid-state packing were missed by those studies. ${ }^{6}$ Recently, we have shown F8BT based PLED gives efficiency numbers beyond the statistical spin limit which cannot solely be explained with a just theoretical upper limit ( $37 \%)$ of DF yield from TTA and need an additional contribution towards singlet generation, which was speculated as TADF in this system. ${ }^{3}$ We demonstrate that F8BT film thickness plays a crucial role in controlling triplet exciton generated DF. Film thickness can influence the thermal activation energy, which governs the triplet's migration through F8BT polymer chains. The quantum chemical calculation suggests thicker films possess lower reorganization energy due to planarization of the polymer chains which helps to achieve enhanced interchain triplet transport. Grazing-incidence wide-angle X-ray scattering (GIWAXS) also indicate significant differences in the mode of molecular packing in thicker films. Therefore, keeping in mind, all the possible sources of excitonic processes and their corresponding time scales, the origin of DF has been studied in solution, film and in electrically excited PLEDs to get a complete insight of highefficiency F8BT PLEDs. Our paper provides a recipe not only for new molecular engineering but also a methodological approach to studying the photophysics of molecular semiconductors.

\section{RESULTS}

\section{DF in electrically excited PLED}

Figure 1a shows the typical chemical structure of F8BT co-polymer system and the corresponding spatial distribution of HOMO (highest occupied molecular orbital) - LUMO (lowest unoccupied molecular orbital) in it. It can be seen (Fig. 1a) that the HOMO level of F8BT is delocalized over the polymer backbone whereas the LUMO is localized in the benzothiadiazole (BT) unit. It is because of the relatively small energy gap between the HOMO levels and larger gap between LUMO levels of the corresponding fluorene (HOMO $\sim 7.76 \mathrm{eV}$, LUMO $\sim 0.18 \mathrm{eV}$ ) and BT (HOMO $\sim-8.42 \mathrm{eV}$, LUMO $\sim-1.38 \mathrm{eV}$ ) units. $^{20}$ Fig. $1 \mathrm{~b}$ shows the absorption and $\mathrm{PL}$ spectra from F8BT solution and film, respectively. The PL in the film is red-shifted from the solution due to the formation of more aggregates in the solid state. ${ }^{21}$ Absolute PL yield of F8BT is in the range of $70 \pm 5 \%{ }^{15}$ and typical outcoupling efficiency estimation is $\sim 20 \%$ in a typical PLED structure. ${ }^{3}$ Hence, the theoretical EQE of F8BT PLED is limited to $3.5 \%(E Q E=1 \times 0.7 \times 0.25 \times 0.2)$. However, in F8BT PLEDs the EQE is significantly controlled by the active layer thickness where a thicker layer $\sim 1.2 \mu \mathrm{m}$ can give EQE far beyond statistical spin limit up to $9 \%{ }^{15}$ and up to $18 \%{ }^{16}$ with enhanced outcoupling. Here we have analyzed state of the art F8BT PLED (film thickness $510 \pm 50 \mathrm{~nm}$ ) (with EQE $\sim 4.6 \%$ and luminous efficiency $\sim 14 \mathrm{~cd} / \mathrm{A}$, Figure S1) with the clear signature of delayed EL in transient EL studies as shown in Fig. 2a. To clarify the role of DF in enhancing PLED efficiency either by TTA or TADF, we record the electrically excited DF after the end of the electrical pulse. A pulse voltage of different pulse heights with a pulse width of $100 \mu \mathrm{s}$ at a frequency of $100 \mathrm{~Hz}$ has been used to excite the PLEDs. The electrically excited DF has often been considered as a result of geminate polaron pair recombination (GP) instead of triplet excitons. $^{22,23}$ We have measured the DF in the presence of negative electrical field to find that electrically excited DF in F8BT PLEDs remains unaffected by negative electric field (see Figure S2). At low applied bias voltage $(\sim 8 \mathrm{~V})$ the transient EL has exponential decay nature which successively changes to powerlaw decay at higher applied bias ( 13 V) (Fig. 2a). In case of higher bias voltage, at the early time, DF decay with slope -0.82 (close to unity) which at longer time delays changes to slope -2.0 . Figure $2 \mathrm{~b}$ shows the electroluminescence (EL) spectrum and the DF spectra for F8BT PLEDs. The DF spectra appear to be similar to the EL (pulse ON time) spectra except little broadening on higher wavelength side. The close spectral match of PF and DF suggests the similar origin for both in electrically excited PLEDs. The little spectral broadening in the case of DF may arise due to spectral diffusion of the triplet excitons in the Gaussian distribution of states in conjugated polymer systems. ${ }^{24}$ To get the correlation 

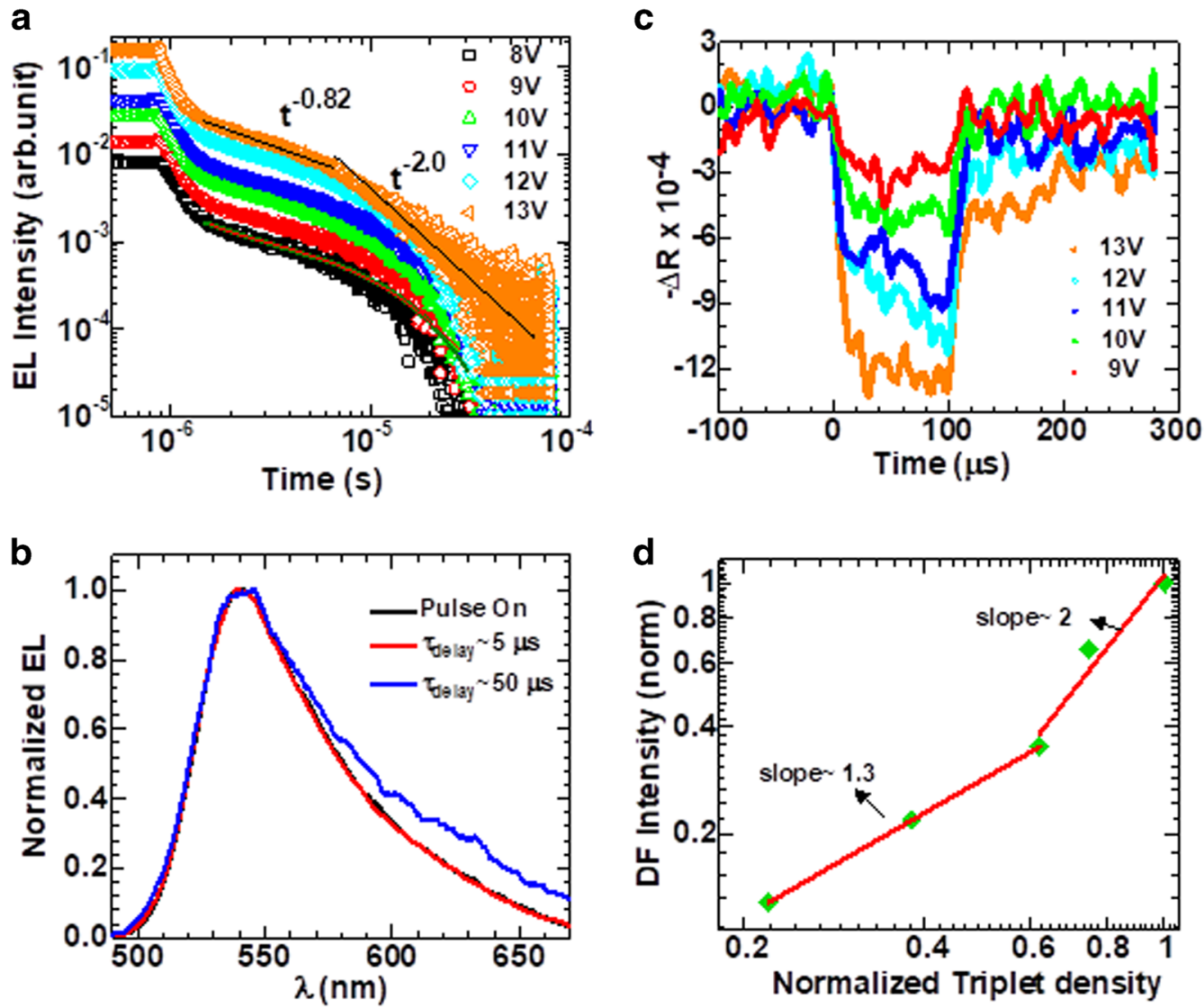

Fig. 2 Delayed Fluorescence under electrical excitation. a Electrically excited time-resolved emission kinetics from F8BT PLED at different bias pulse voltages. At lower voltages, the transient EL decay is exponential which changes to power-law decay at higher applied bias and can be fitted with $\sim t^{-0.82}$ power law which changes to $\sim t^{-2.0}$ at longer times. $\mathbf{b}$ EL spectra during electrical pulse ON time and delayed emission with a time delay of 5 and $50 \mu \mathrm{s}$ in F8BT PLED. c Triplet absorption signal from F8BT PLEDs is captured by probing the device at $\lambda=780 \mathrm{~nm}$ (close to $\mathrm{T}_{1}-\mathrm{T}_{n}$ absorption peak maxima). d Normalized DF intensity vs. normalized triplet density (derived from c)

between electrically excited DF with triplet excitons, we have used transient absorption spectroscopy on PLEDs at different applied pulsed voltages. The triplet exciton has been probed at a wavelength of $780 \mathrm{~nm}$ (close to $\mathrm{T}_{1}-\mathrm{T}_{n}$ absorption maxima in F8BT). ${ }^{25}$ The corresponding change in reflected beam has been captured to determine triplet population density in operational PLED. Figure 2c shows the triplet absorption signal of F8BT PLEDs. It can be noticed that triplet absorption gets enhanced with an increase in the height of applied bias voltage. Figure $2 \mathrm{~d}$ shows the normalized DF intensity at $1 \mu$ s delay under electrical excitation concerning electrically generated normalized triplet density. Triplet density is derived from Fig. $2 \mathrm{c}$ using $\Delta R=N_{T} \sigma_{T} d$, where $N_{T}$ is the triplet density, $\sigma_{T}$ is the triplet absorption cross section $\sim 2.6 \times 10^{-16} \mathrm{~cm}^{-2}$ estimated from triplet absorption ${ }^{3}$ and $d$ is the active layer thickness. The DF intensity initially varies linearly with triplet density after which it enhances as a square of triplet excitation populations.

\section{DF in F8BT solution and solid film at room temperature}

To record the long-lived DF in F8BT solution, we use variable gatedelay TRES technique, ${ }^{26,27}$ to capture the total decay kinetics (PF and DF) from $1 \mathrm{~ns}$ to $\sim 1 \mathrm{~ms}$ with an overall intensity drop over 13 orders of magnitudes. Figure $3 a$ shows the complete decay kinetics of F8BT solution (with $\sim 10^{-4} \mathrm{M}$ concentration in a quartz cuvette sealed in the $\mathrm{N}_{2}$ atmosphere) excited at a wavelength of $355 \mathrm{~nm}$ with energy density $\sim 527 \mu \mathrm{J} / \mathrm{cm}^{2}$ at room temperature in $p$-xylene as solvent media. We have also done the DF measurement as a function of solvent polarity as shown in Figure S3a and $\mathrm{S} 3 \mathrm{~b}$ for F8BT solution in Chlorobenzene and o-dichlorobenzene.
The inset of Fig. 3a shows the decay kinetics of prompt fluorescence $(\mathrm{PF})$ with lifetime $\left(\tau_{\mathrm{PF}}\right) \sim 2.28 \mathrm{~ns}$ obtained by monoexponential fitting. In F8BT solution (irrespective of solvent polarity), there is a clear transition from PF to DF region at $\sim 10$ 's ns. In solution, the DF consists of three distinct luminescence decaying time regions. Region $A$ has a faster exponential decay nature (which will be called DF1 now onwards) whereas region $B$ has much slower exponential decay (which will be called DF2 from here onwards). Both DF1 and DF2 have bi-exponential decay. ${ }^{12}$ Both DF1 and DF2 originate from triplet excitons, which can be correlated with their non-linear dependence on the prompt fluorescence intensity (DF vs PF slope $\sim 1.5$ ). Such, multiexponential nature of decay may arise from different spatial distribution or anisotropic character of packing of polymer chains. $^{28}$ The third part of the delayed fluorescence region, i.e., region $C$ (will be called DF3 here onwards) deviates from the exponential nature and follows a power law decay instead. ${ }^{29}$ The order of DF3 magnitude is much lower (lower by $10^{-11}$ order magnitude), and its contribution can be neglected in the present study. The DF life has been observed to be increased in higher polarity solvent due to the enhanced relaxation time of the charge transfer state (Table S1). It is also seen that the DF decay much faster in case of an aerated solution in the presence of oxygen thus indicating triplet excitons as the cause of DF generation (Figure S4). Figure $3 \mathrm{~b}$ shows time integrated DF1 (time delay $\sim 200 \mathrm{~ns}$ ) and DF2 (time delay $\sim 10 \mu \mathrm{s}$ ) concerning PF intensity. In both the cases (for DF1 and DF2) a slope between 1 and 2 has been observed. The DF spectra in F8BT solution also matches with the PF except for a bit broader FWHM compared to PF spectrum 
a
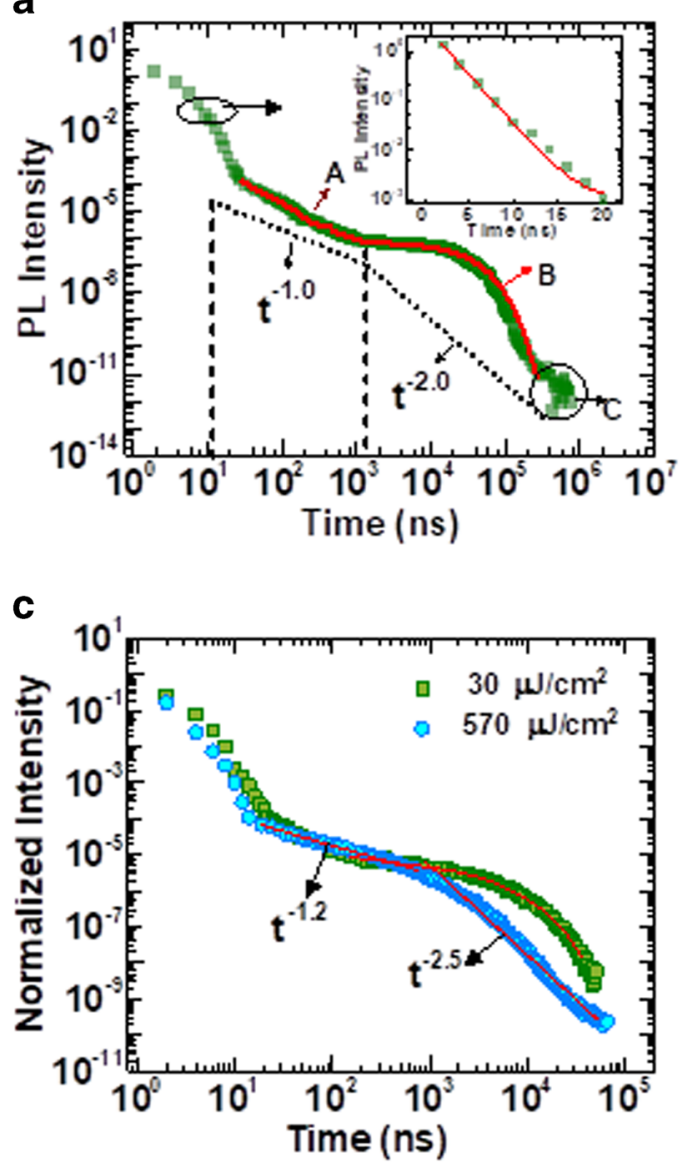

b

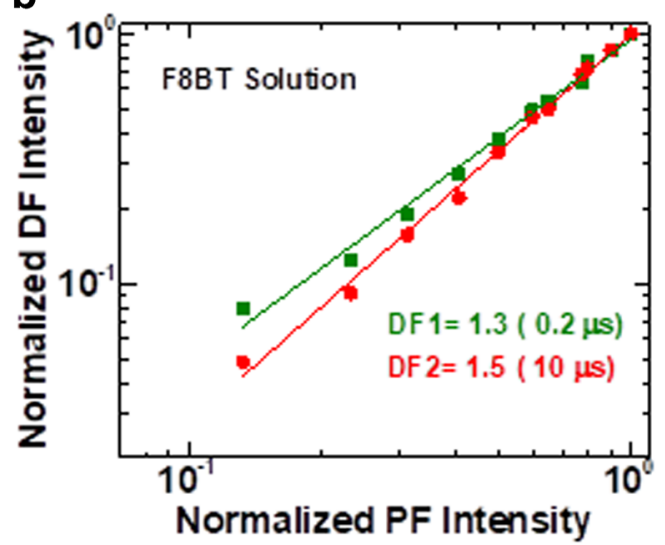

d

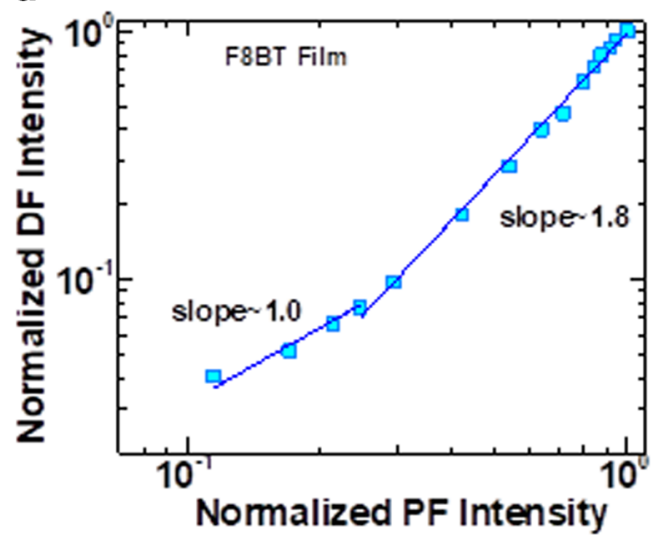

Fig. 3 Time resolved photoluminescence at room temperature. a Time-resolved emission decay of F8BT solution in $p$-xylene. The inset shows time-resolved PF in F8BT solution. The dotted line is a guide to the eye to show the nature of power-law decay. The solid red line is biexponential fit to experimental data for DF1 and DF2. b Time-integrated DF vs. PF intensity derived for F8BT solution at DF1 (at time delay $\sim 200 \mathrm{~ns}$ ) and DF2 (at time delay 10 $\mu \mathrm{s}$ ) regions, respectively, the solid line is linear fit to data for different fluence range. c Time-resolved emission decay in F8BT neat solid film containing low $\left(30 \mu \mathrm{J} / \mathrm{cm}^{2}\right.$, solid green square) and high $\left(570 \mu \mathrm{J} / \mathrm{cm}^{2}\right.$, solid blue circle) laser fluence. At low fluence, DF decays exponentially (red solid line) whereas at high fluence it has power law decay (red solid line). d The time-integrated PF and DF intensity vs. laser fluence plots for F8BT solid film, linearly fitted with dual slopes

Table 1. Room temperature average triplet lifetimes, broadening of $\mathrm{PF}$, DF emission spectra and effective triplet activation energy parameters of F8BT as a function of film thickness

\begin{tabular}{lllll}
\hline $\begin{array}{l}\text { Film } \\
\text { thickness } \\
(\mathrm{nm})\end{array}$ & $\begin{array}{l}<0 \mu \mathrm{J} / \mathrm{cm}^{2} \\
(\mu \mathrm{s})\end{array}$ & $\begin{array}{l}\text { FWHM (PF) } \\
(\mathrm{meV} / \mathrm{nm})\end{array}$ & $\begin{array}{l}\text { FWHM } \\
(\mathrm{DF}) \\
(\mathrm{meV} / \mathrm{nm})\end{array}$ & $\begin{array}{l}\text { Effective } \\
\text { activation } \\
\text { energy }(\mathrm{meV})\end{array}$ \\
\hline 110 & 0.73 & $287 / 62$ & $309 / 73$ & $179 \pm 31$ \\
310 & 1.8 & $251 / 56$ & $255 / 59$ & $108 \pm 8$ \\
560 & 2.4 & $204 / 46$ & $240 / 55$ & $86 \pm 11$ \\
\hline
\end{tabular}

(see Figure S6d to S6f), similar to the case of PLEDs (Fig. 2b). Figure $3 \mathrm{c}$ shows the time-resolved PL decay kinetics for F8BT solid neat film $(\sim 310 \mathrm{~nm})$ at low $\left(\sim 30 \mu \mathrm{J} / \mathrm{cm}^{2}\right)$ and high fluence $(\sim 570 \mu \mathrm{J} /$ $\mathrm{cm}^{2}$ ), respectively. ${ }^{3}$ It can be observed that the DF decays much faster in the solid film compared to the solution (Fig. 3a). At low fluence, however, the DF intensity follows tri-exponential emission decay with an average DF lifetime $\sim 1.8 \mu$ s which are observed to be increased with film thicknesses (see Figure S7, Table 1). It is observed that the non-exponential contribution increases in the DF decay with the increase in the film thickness, even at lower fluence (Fig. 3c, Figure S7a-S7b). At high fluence, the DF can be subdivided into two main decay regions in a neat F8BT solid film in contrast to a solution where we have three regions. The first decay region (region A: DF1s) follows a power law decay $\left(\sim t^{-1.2}\right)$ region between $200 \mathrm{~ns}$ to $1 \mu \mathrm{s}$ timescale which subsequently changes to $\sim t^{-2.5}$ decay in region B (DF2s) up to $20 \mu \mathrm{s}$. At higher fluence, the DF also decay much faster due to an enhanced bimolecular process for all the film thicknesses (see Figure S7). The transition from near unity slope to the cubic slope (suggesting dispersive to non-dispersive triplet kinetics) ${ }^{26,27}$ occurs at a lower time for thinner films. In the case of $\sim 110 \mathrm{~nm}$ film (Figure S7a), the initial power-law decay changes from a slope $1.4-3$ at $\sim 2.5 \mu \mathrm{s}$, whereas, for $\sim 310 \mathrm{~nm}$ (Fig. 3c) film power law changes from $t^{-1.3}$ to $t^{-2.7}$ at $3.5 \mu \mathrm{s}$, and for $\sim 510 \mathrm{~nm}$ (Figure S7b) power-law decay transition from $t^{-1.2}$ to $t^{-3.0}$ occurs even at a longer time $\sim 4 \mu \mathrm{s}$. This turn over time $\left(t_{r}\right)$ is related to the density of state (DOS) broadening $(\sigma)$ via $t_{r}=(1 / v) \times \exp \left[\left(-\sigma / k_{\beta} T\right)^{2}\right]$ where $v$ is jump frequency for excitons, $k_{\beta} T$ is the available thermal energy. ${ }^{29}$ Fig. $3 d$ shows normalized PF intensity vs. normalized DF intensity, and we note that it fits with dual slopes. Initially, at lower fluence $\left(100 \mu \mathrm{J} / \mathrm{cm}^{2}\right)$, PF vs. DF intensity follows a linear ${ }^{17}$ relation and then it gets almost quadratic. ${ }^{23}$ In case of all the solid films, PF and DF spectra closely match with each other (see Figure S8).

Temperature-dependent DF in F8BT solid film

Triplet exciton transport through conjugated polymer chains effectively controls the DF yield. To understand triplet energy, 

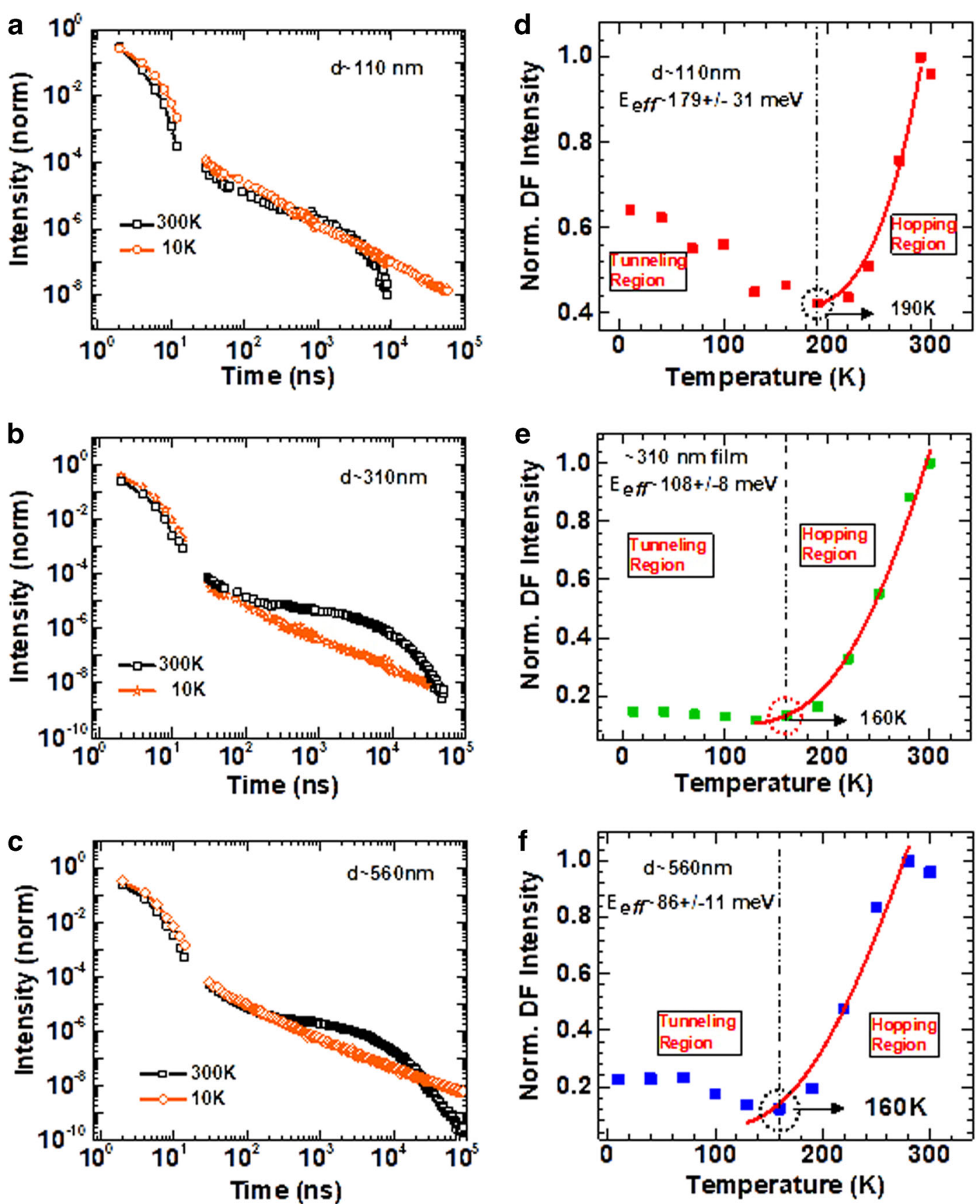

Fig. 4 Temperature-dependent DF dynamics. Temperature-dependent time-resolved PL decay for F8BT films of different thicknesses. Each decay curve has a fast and a slow component. a For thinnest film $(\sim 110 \mathrm{~nm})$ the DF has almost remained unaffected relative to temperature, whereas for $\mathbf{b}$ intermediate thickness $(\sim 310 \mathrm{~nm})$ and $\mathbf{c}$ thickest film $(\sim 560 \mathrm{~nm})$ studied, the DF decay dynamics change from exponential to power-law decay at lower temperatures. Time-integrated DF intensity relative to temperature for $\mathbf{d} 110 \mathrm{~nm}$ e $310 \mathrm{~nm}$ and f $560 \mathrm{~nm}$-thick films. The DF intensity has two different temperature dependence namely: thermally assisted hopping region and weakly temperature-dependent single step tunneling regions

transfer in F8BT film having different thickness, we have performed temperature-dependent time-resolved PL decay. Figure $4 \mathrm{a}-4 \mathrm{c}$ shows the temperature-dependent time-resolved $\mathrm{PL}$ decay for $110 \mathrm{~nm}, 310 \mathrm{~nm}$, and $560 \mathrm{~nm}$ thick F8BT solid films at excitation fluence $\sim 30 \mu \mathrm{J} / \mathrm{cm}^{2}$. The energy density is kept low to minimize photo-oxidation due to long duration laser exposure. For the $110 \mathrm{~nm}$ film, time-resolved PL decay changes from exponential to power-law nature as the temperature goes down. However, the relative difference in decay rates at different temperature remains almost unchanged (Figure S9a). For thicker films (i.e., $310 \mathrm{~nm}$ and $560 \mathrm{~nm}$ ) on the other side, the temperature effect is strongly visible. For $310 \mathrm{~nm}$ (Fig. $4 \mathrm{~b}$ and Figure S9b) and $560 \mathrm{~nm}$ (Fig. 4c, Figure S9c) films, the TRES decays exponentially at room temperature $(300 \mathrm{~K})$. With further reduction in temperature (from $300 \mathrm{~K}$ to $10 \mathrm{~K}$, see in Figure S8), the exponential decay slowly transits into the power law nature. The time-integrated DF intensity vs. temperature for all film thicknesses shows an initial decrease in DF intensity (captured at $200 \mathrm{~ns}$ time delay with $800 \mathrm{~ns}$ 
Table 2. Variation in the reorganization energy $(\lambda)$ and activation energy $\left(E_{\mathrm{a}}\right)$ with respect to the dihedral angle $(\Phi)$ between the donor and the acceptor moieties of F8BT

\begin{tabular}{|c|c|c|c|c|c|}
\hline$E$ & -50840.4228 & -50840.4166 & -50840.4054 & -50840.3898 & -50840.3714 \\
\hline$E^{*}$ & -50840.2831 & -50840.3224 & -50840.3114 & -50840.2979 & -50840.2812 \\
\hline$\lambda_{1}$ & 0.1397 & 0.0942 & 0.0940 & 0.0919 & 0.0902 \\
\hline$E_{+}$ & -50833.4126 & -50833.3806 & -50833.3813 & -50833.3781 & -50833.3707 \\
\hline$\lambda$ & 0.2859 & 0.1905 & 0.1858 & 0.1811 & 0.1768 \\
\hline$E_{\mathrm{a}}=\lambda / 4$ & 0.0714 & 0.0476 & 0.0464 & 0.0452 & 0.0442 \\
\hline
\end{tabular}

integration time, Figure S10) with lowering the temperature below $300 \mathrm{~K}$ and reaches to an absolute minimum point which is also film-thickness-dependent. Figure $4 \mathrm{~d}$ shows the integrated DF intensity vs. temperature wherein the low-temperature region (after the minimum DF intensity) rises slowly with further reduction in temperature. For $310 \mathrm{~nm}$ (Fig. 4e) and $560 \mathrm{~nm}$ film (Fig. 4f) increase of DF intensity in the low-temperature regime, is relatively less temperature-dependent. It indicates two competing processes are generating DF in this polymer system, which will be discussed in detail afterward.

\section{Solid-state molecular packing}

To probe differences in molecular packing in thin and thick films, GIWAXS measurements have been performed, see Figure S12. The $110 \mathrm{~nm}$ thick film exhibits well-defined reflections characteristic of the three-dimensionally ordered packing of polymer chains, as reported previously. ${ }^{30}$ With increasing thickness the most obvious change is an increase in the degree of 'arcing' of peaks indicating that in thicker films the crystallites possess a larger range of orientations with respect to the substrate. This is to be expected since in a thin film there is greater registry between crystallites and the substrate while with increasing film thickness the influence of the film interfaces is diminished as it approaches a bulk sample. On closer inspection of the scattering patterns, however, the thicker films exhibit a different set of peaks which are not found in the thin film sample. This new packing geometry matches that previously seen for Donley et al. $^{30}$ for lower molecular weight samples of F8BT which has furthermore been associated with a different F8BT packing structure where in adjacent chains F8 units cofacially packing adjacent to BT units resulting in an 'alternating structure.' In this alternating structure, the BT unit also has a lower dihedral angle with respect to the F8 unit compared to the structure where F8 (BT) units cofacially pack next to F8 (BT) units in adjacent chains. Thus the GIWAXS measurements provide direct evidence that thin and thick films have different molecular packing geometries, and that these different molecular packing geometries are likely to possess different dihedral angles.

Due to change in molecular packing we have observed a change in photoluminescence decay time. An increase in the prompt fluorescence lifetime with thickness has been observed in time correlated single photon counting (TCSPC) measurement (Figure S5, Table S1.2). There is a possibility that the molecular packing may also alter the dipole orientation which may affect the out-coupling efficiency for PLQY as well as charge carrier balance factor in operational PLEDs. However, we confine ourselves in this study to the variation in singlet yield due to thickness dependent triplet exciton dynamics.
Quantum chemical calculation

The polymer film morphology dependent triplet transfer process has been further verified by performing density functional theory (DFT)-based calculations of the reorganization energy $(\lambda)$ as a function of dihedral angle $(\Phi)$ between the donor (F8) and the acceptor moiety (BT) of a F8BT molecule (the related computational details are given in the Section S.2.1 of the SI). For this purpose, we first relaxed the F8BT molecule to obtain its ground state geometry (Figure S14). The optimized dihedral angle between the donor and the acceptor units of a F8BT molecule is found to be $\sim 37^{\circ}$. However, this angle is known to reduce with the increase in interchain interactions, ${ }^{31}$ or with a change in molecular packing structure such as observed by GIWAXS. Since, in bulk F8BT, the dihedral angle reduces to $\sim 14^{\circ}$ by strong interchain interactions, ${ }^{31}$ we argue that the dihedral angle between the ' $\mathrm{F} 8$ ' and 'BT' units will decrease on going from gaseous to the solution to thin film phase. Further in a film, $\Phi$ should reduce with the increase in film thickness (GIWAXS Figure S12 and PL FWHM Figure S8). We, therefore, subsequently reduced $\Phi$ from $37^{\circ}$ to $15^{\circ}$ by manually fixing the dihedral angle and allowed the rest of the molecule to relax, to study the variation in $\lambda$ concerning $\Phi$. It is evident from the data tabulated in Table 2 that the reorganization energy decreases monotonically with the decrease in the dihedral angle, as expected. ${ }^{32}$

\section{Discussions}

There can be many underlying processes in generating DF in conjugated polymers where we need to differentiate triplet-triplet annihilation (TTA), ${ }^{29}$ thermally activated delayed fluorescence (TADF) and geminate polaron pairs (GP) recombination. ${ }^{9}$ Positive pulse followed by negative bias experiment ruled out GP recombination as the origin of DF (Figure S2). Hence, there is the role of triplet excitons in the generation of DF. For TTA to be dominated, DF intensity varies quadratically concerning triplet density, whereas for RISC generated DF, it is linear with respect to triplet density. ${ }^{33}$ In case of electrically excited DF at the lower bias case mono-molecular triplet decay rate is faster $\left(k_{T}, k_{R I S C}>k_{T T}\right)$ compared to the bi-molecular rate of TTA $\left(k_{T}\right)$, which causes exponential decay of DF emission. At higher bias case, enhanced triplet exciton density causes power law decay due to $\Pi A$, where DF decays with slope -0.82 due to dispersive triplet exciton migration (where TAA decay rate $\left(k_{T}\right)$ is time-dependent) ${ }^{26}$ which subsequently changes to slope -2.0 due to non-dispersive triplet exciton transport (where $k_{\pi}$ no longer remains time-dependent). ${ }^{26,29}$ From Fig. $2 d$, it is clear that at a lower bias voltage (up to $11 \mathrm{~V}$ ) the DF intensity increases linearly whereas at a higher bias voltage (above $11 \mathrm{~V}$ ) it grows in a quadratic fashion relative to triplet exciton density. If we try to fit the same data (Fig. 2c) with a 
single slope, it appears as 1.3, suggesting the existence of a mixed mono and bi-molecular processes. In case of the optically excited F8BT solution also, the PF vs. DF emission integral shows a slope of $\sim 1.5$ (Fig. 3b) which means the presence of bi-molecular process alongside with mono-molecular originated delayed emission (irrespective of solvent polarity as seen in Figure S6a -S6c). In case of F8BT solid film, the DF intensity increases linearly with PF until $100 \mu \mathrm{J} / \mathrm{cm}^{2}$ and then it grows quadratically for higher fluence. It suggests the presence of mono-molecular DF origin at low fluence and bi-molecular process induced DF at high fluence. For differentiating the possible presence of RISC conventional TADF mechanism ${ }^{34}$ in F8BT PLEDs from dominant TTA process, ${ }^{3,6}$ we have performed magnetic field modulated electroluminescence (MEL, Figure S11) study on F8BT PLEDs ${ }^{10,35}$ since both RISC and TTA are highly spin-dependent. Low $(<40 \mathrm{mT})$ and high magnetic field $(>4 \mathrm{mT})$ regimes have different impact on (via modifying hyperfine coupling (HFC)-mediated intersystem crossing process) the RISC generated TADF and TTA generated DF. ${ }^{35}$ The dominance of TADF is effectively controlled via the successful transition from triplet charge transfer states $\left({ }^{3} \mathrm{CT}\right)$ to singlet charge transfer states $\left({ }^{1} \mathrm{CT}\right) .^{34}$ Under external magnetic field of low strength (usually below $40 \mathrm{mT}$ ), the triplet states degeneracy breaks down due to Zeeman splitting, resulting into three subsequent triplet sub-levels $T_{+1}, T_{0}$, and $T_{-1} \cdot{ }^{34}$ This leaves only one channel $T_{0}$ for RISC thus reducing the overall EL intensity. ${ }^{35}$ On the contrary in case of TTA dominated system at the low magnetic field regime overall EL intensity shows an initial positive rise which subsequently decays down at the high magnetic field. $^{36-38}$ In PLEDs under electrical injection, polaron pair forms before the formation of excitonic species. The ISC from singlet to triplet polaron pair states gets restricted under the influence of low strength magnetic field via modulating the HFC strength between the singlet and triplet polaron pair states. This results in a positive MEL intensity at the low field regime. ${ }^{37}$ At the high field regime, the magnetic field restricts the spin-flipping of the singlet character of $\Pi$ pair (which is responsible for $\Pi$ TA generated singlet excitons), henceforth reducing the emission intensity. ${ }^{37}$ We have checked MEL response $(=\mathrm{EL}(\mathrm{B})-\mathrm{EL}(0) / \mathrm{EL}(0))$ of F8BT PLEDs with different emissive layer under constant current injection mode (to avoid magneto conductance induced change in EL intensity) ${ }^{38}$ (see in Figure S11). For all the emissive layer thicknesses we have observed positive MEL response intensity at low injection current levels which tends to fall at the external magnetic field levels exceeding $50 \mathrm{mT}$ (as described above). At a high injection, current level MEL decays faster with increasing external magnetic field. The MEL response of F8BT PLEDs suggests that TTA dominates the DF generation. It is needed to be mentioned here that since the $\mathrm{HFC}$ field mediated negative response (in case of ${ }^{3} \mathrm{CT} \rightarrow{ }^{1} \mathrm{CT}$ state mediated TADF process) happens within few $\mathrm{mT}(\sim 4.5 \mathrm{mT})$, it is hard to untangle from the underneath dominate TTA process.

Now the question arises how the TTA generated DF governs the efficiency enhancement of F8BT PLEDs with merely increasing the polymer film thicknesses. Triplet exciton diffusion and lifetime can be significantly altered as a function of temperature in films of different polymer packing. Triplet energy transport in the disordered polymeric system occurs via a multi-step hopping mechanism in the high-temperature regimes whereas at low temperature it follows single step tunneling mechanism. ${ }^{39}$ Triplet excitons migrate following the Dexter energy transfer mechanism involving simultaneous electron exchange from neighboring molecule's HOMO and LUMO. The corresponding electron transfer rate can be expressed with the help of Marcus theory: ${ }^{14}$

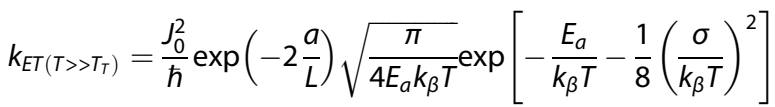

where $J_{0}$ is the electronic coupling strength between neighboring molecules, a is average distance between two molecular sites, $L$ is the triplet exciton localization radius, $E_{\mathrm{a}}$ is the activation energy required for hopping, and $\lambda$ is the reorganization energy. By considering the energetic disorder parameter $\sigma$, the effective activation energy $\left(E_{\text {eff }}\right)$ for triplet exciton transport becomes $E_{\text {eff }}=E_{a}+\frac{\sigma^{2}}{8 k_{\beta} T}$. Thus effective activation energy is a signature of polymer film's disorder. As it is clear from our temperaturedependent TRES decay, in F8BT films (as a function of polymer film thickness) triplet excitons transport is significantly governed via the polymer chain packing. For the thinnest film $(\sim 110 \mathrm{~nm})$ studied here, we observed DF decay dynamics remains almost temperature invariant and follows the $t^{-1.3}$ law. On the other side for thicker films studied here (such as for $310 \mathrm{~nm}$ and $560 \mathrm{~nm}$ film thicknesses) DF decay dynamic slowly changes from exponential to power law decay. The temperature-dependent DF intensity as a function of film thickness shows us some crucial points, for all the film thicknesses studied there are two distinct temperature regions: (1) strong temperature-dependent region and (2) weak temperature-dependent region. ${ }^{13}$ The effective activation energy of triplet transport has been determined by fitting the hightemperature region with the equation $l_{D F}=$ $A \sqrt{1 / T} \exp \left(-E_{\text {eff }} / k_{\beta} T\right)+B\left(\right.$ where $\mathrm{A}=\frac{J_{0}^{2}}{\hbar} \exp \left(-2 \frac{a}{L}\right) \sqrt{\frac{\pi}{4 E_{a} k_{\beta}}}$ and $\mathrm{B}$ is an offset parameter relative to the DF intensity) having a similar form as Eq. 1, depicting triplet energy transport between two neighboring molecules (thus can be considered as proportional to TTA rate constant $\mathrm{k}_{\mathrm{T}}$ ). ${ }^{12}$ The $E_{\text {eff }}$ is highest for the thinnest films $\sim 179 \pm 31 \mathrm{meV}$, and lowest $\sim 86 \pm 11 \mathrm{meV}$ for the thickest film studied here. As mentioned earlier in this paragraph $E_{\text {eff }}$ is a measure of disorder parameter of the film. Hence thinnest film possesses a higher disorder as compared to thicker films. It demands a narrower emission profile in the more ordered F8BT films which is supposed to be here the thicker ones. Figure S8a to S8C shows that both PF and DF emission are narrower in thick films compared to thinner films. Table 1 depicts the derived experimental FWHM for PF and DF emission along with determined triplet activation energies. The weak temperature dependent regions on the other side can be explained with the help of single step tunneling process in the light of Miller-Abraham model where the triplet energy transport between two neighboring molecules can be expressed as, ${ }^{14}$

$K_{E T\left(T<T_{T}\right)}=U_{0} \exp \left(-2 \frac{a}{L}\right) \exp \left(-\frac{1}{2}\left(\frac{\sigma}{k_{\beta} T}\right)^{2}\right)$

Equation 2 indicates that in low-temperature regime also triplet transport is controlled via polymer film's disorder parameter. Hence as observed in the case of $\sim 110 \mathrm{~nm}$ film in the lowtemperature regime the slow rise of DF intensity is due to possession of the higher disorder as compared to the thicker films. The transition temperature from multistep hopping region to single step tunneling region is high $\left(T_{\mathrm{T}} \sim 190 \mathrm{~K}\right.$ for $110 \mathrm{~nm}$ film and $T_{\mathrm{T}} \sim 160 \mathrm{~K}$ for the $560 \mathrm{~nm}$ film) for thinner films due to the presence of more disorder. ${ }^{39}$ Thus after elaborately analyzing the temperature-dependent TRES kinetic in F8BT films of different thicknesses, it is evident that thermally activated triplet hopping is the dominant mechanism for enhancing the singlet yield. This thermally-assisted triplet hopping is strongly an interchain transport since it is absent in the F8BT in dispersive media (see Figure S13a-S13b). The uniqueness of the thermally-assisted triplet transport as a function of F8BT polymer film differs from the polyfluorene (PFO) system where high temperature thermally activated TTA is absent (see Figure S13d-S13f). As described earlier, F8BT can also form an alternating structure of polymer chains where adjacent neighboring polymer chains are shifted from each other in such a way that the 'F8' donor unit in one chain faces the acceptor 'BT' unit of the neighboring chain. ${ }^{30}$ It makes 
the interchain energy transfer process strong in the case of the F8BT system.

Our DFT calculations also qualitatively agree with the experimental results and justify the conclusion made above from the measured data, where we have observed a reduction in the activation energy which depends directly on $\lambda$ (Table 2), ${ }^{13}$ and increase in triplet exciton transportation with the increase in film thickness (which possesses lower dihedral angle as also evident from the GIWAXS study). Furthermore, this is well reported in the literature that for efficient electron-light conversions, a photophysical phenomenon like TTA plays a significant role when $\Delta \mathrm{E}_{\mathrm{ST}}$ increases and the energy of two triplet excitons are close to or larger than one singlet exciton, i.e., when $\mathrm{E}_{\mathrm{T} 1} / \mathrm{E}_{\mathrm{S} 1}$ is close but higher than 0.5..$^{40,41}$ Therefore, to furthermore strengthen our judgment we also calculated the lowest singlet $\left(E_{S 1}\right)$ and triplet $\left(E_{T 1}\right)$ excited states energy, and the respective singlet-triplet splitting energy $\left(\triangle \mathrm{E}_{\mathrm{ST}}\right)$ for F8BT molecules with a different dihedral angle $\left(15^{\circ}, 20^{\circ}, 25^{\circ}, 30^{\circ}, 37^{\circ}\right.$, and $\left.50^{\circ}\right)$. Though the variations are small, it is still clearly evident from Table S2 in SI that with the decrease in the dihedral angle (i.e., in thicker films), $\Delta \mathrm{E}_{\mathrm{ST}}$ increases and $\mathrm{E}_{\mathrm{T} 1} / \mathrm{E}_{\mathrm{S} 1}$ decreases to approach towards 0.5 value, giving a clear indication of escalation of TTA process in thicker films.

\section{CONCLUSIONS}

In summary, F8BT polymer system gives efficiency number beyond spin statistics in PLEDs where the role of triplet excitons has been studied in detail as a function of film thickness, excitation fluence, magnetic-field, and temperature. We have studied the DF emission properties in F8BT co-polymer system in solution, solid films under optical excitation and in F8BT PLEDs under electrical excitation along with a structural study by GIWAXS and quantum chemical calculations to understand the possible role of triplet excitons. Our temperature-dependent TRES kinetic as a function of polymer film thickness suggests it is the thermally activated TTA which controls the DF yield in the F8BT emissive layer. This thermally activated TTA is strongly dependent on interchain ordering. This nature of triplet transport in F8BT polymer system provides the guidelines for molecular engineering, where efficiency number can be controlled significantly by facilitating the dominating triplet fusion process as a function of film thickness or packing of chains. Thicker films based PLEDs are preferred for a technological point of view to increase the device yield.

\section{MATERIALS AND METHODS}

All solution based samples have been prepared in anhydrous solvents inside the nitrogen filled glove box. The F8BT polymer is supplied by Cambridge display Technology (CDT). The dilution of the F8BT solution is kept constant at $\sim 10^{-4} \mathrm{M}$. The solution is filled in $1 \times 1 \mathrm{~cm}$ nitrogen sealed quartz cuvette. Neat F8BT solid films have been prepared by spin coating on quartz substrate from F8BT solution containing different polymer concentration to get film thickness $\sim 110 \mathrm{~nm}, 310 \mathrm{~nm}$ and $\sim 560 \mathrm{~nm}$, respectively. PLEDs are fabricated on ITO coated glass substrate with $\mathrm{ZnO} /$ PEIE as electron injection layer, F8BT as emissive layer $(\sim 560 \mathrm{~nm})$ and $\mathrm{MoO}_{3} / \mathrm{Au}$ as a hole injecting contact. All samples (both solution and neat solid films) are optically excited via the third harmonic of Nd-YAG Laser (Innolas piccolo AOT) at $3.49 \mathrm{eV}$ with a repetition rate of $1 \mathrm{kHz}$ and pulse width $\sim 840 \mathrm{ps}$. The time-resolved emission spectra (TRES) has been detected with the help of Andor-gated intensified CCD and integrated with Shamrock spectrometer (303i) by using variable gate delay technique ${ }^{1}$ record an intensity drop up to 13 order of magnitude from ns to $1 \mathrm{~ms}$ in the timescale. The PLEDs are electrically excited by a pulse voltage (from Lecroy Arbitrary fuction generator) of width $100 \mu \mathrm{s}$ at a repetition rate of $100 \mathrm{~Hz}$, and the time-resolved electroluminescence was captured using an avalanche photodetector of rising time $\sim 10 \mathrm{~ns}$ and detected the trace on a $1 \mathrm{GHz}$ oscilloscope by Tektronics. For negative bias measurement, the devices are excited with a negative bias of height $6 \mathrm{~V}$ immediate after the end of a positive pulse (from Lecroy Arbitrary fuction generator) to remove the delayed emission generated via trapped charge carriers. For low temperature $(\sim 10 \mathrm{~K})$ TRES under optical excitation, the F8BT solid film was placed on the cold finger of a closed cycle Helium cryostat (Janis CCS$300 \mathrm{~S} / 204$ ) under a dynamic vacuum of $\sim 10^{-5} \mathrm{mbar}$. For magneto electroluminescence measurement the devices are kept in between the magnetic poles such that the current flowing through the devices remains perpendicular to the applied magnetic field which ensures maximum applied field strength. The devices are operated at the constant current mode using the Keithley 2400 source meter to avoid the effect of magnetoconductance. The EL emission is collected using an optical fiber and therefore, detected using Shamrock spectrometer integrated with ICCD with 50 ms integration time. The modulated EL response (MEL) has been determined via $\operatorname{EL}(\mathrm{B})-\mathrm{EL}(0) / \mathrm{EL}(0)$, where $\mathrm{EL}(\mathrm{B})$ and $\mathrm{EL}(0)$ are the $\mathrm{EL}$ emission in the presence and absence of an external magnetic field, respectively. The magnetic field is measured using a Hall probe Gauss meter placed near to the PLEDs.

\section{DATA AVAILABILITY}

The data that support the findings of the present study are available from the authors on reasonable request; please see author contributions for specific data sets.

\section{ACKNOWLEDGEMENTS}

We thank Cambridge Display Technology (CDT), Ltd., for supplying the F8BT. This work was partially supported by DST-AISRF. We also acknowledge the support of the NCPRE for the device fabrication facility and the School of Natural Sciences, Shiv Nadar University for providing the high-performance computing facility to perform all calculations. Part of this work was performed at the SAXS/WAXS beamline at the Australian Synchrotron, part of ANSTO. A.D. acknowledges the UGC for a research fellowship.

\section{AUTHOR CONTRIBUTIONS}

Project planning was done by A.D and D.K. Project designing, and data analysis was done by A.D., A.R., and D.K. GIWAX study has been done by N.C. and C.R.M. Computational study has been done by D.C. and P.J.

\section{ADDITIONAL INFORMATION}

Supplementary information accompanies the paper on the npj Flexible Electronics website (https://doi.org/10.1038/s41528-018-0042-0).

Competing interests: The authors declare no competing interests.

Publisher's note: Springer Nature remains neutral with regard to jurisdictional claims in published maps and institutional affiliations.

\section{REFERENCES}

1. Tang, C. W., VanSlyke, S. A. \& Chen, C. Electroluminescence of doped organic thin films. J. Appl. Phys. 65, 3610-3616 (1989).

2. Burroughes, J. et al. Light-emitting diodes based on conjugated polymers. Nature 347, 539-541 (1990).

3. Dey, A., Rao, A. \& Kabra, D. A complete quantitative analysis of spatio-temporal dynamics of excitons in functional organic light-emitting diodes. Adv. Opt. Mater. (2016).

4. Giebink, N. C. \& Forrest, S. R. Quantum efficiency roll-off at high brightness in fluorescent and phosphorescent organic light emitting diodes. Phys. Rev. B 77, 235215 (2008)

5. Kondakov, D. Y., Pawlik, T. D., Hatwar, T. K. \& Spindler, J. P. Triplet annihilation exceeding spin statistical limit in highly efficient fluorescent organic lightemitting diodes. J. Appl. Phys. 106, 124510 (2009).

6. Wallikewitz, B. H., Kabra, D., Gélinas, S. \& Friend, R. H. Triplet dynamics in fluorescent polymer light-emitting diodes. Phys. Rev. B 85, 045209 (2012).

7. Kondakov, D. Y. Triplet-triplet annihilation in highly efficient fluorescent organic light-emitting diodes: current state and future outlook. Phil. Trans. Royal Soc. Lond. A Math. Phys. Eng. Sci. 373, https://doi.org/10.1098/rsta.2014.0321 (2015).

8. Goushi, K., Yoshida, K., Sato, K. \& Adachi, C. Organic light-emitting diodes employing efficient reverse intersystem crossing for triplet-to-singlet state conversion. Nat. Photon. 6, 253-258 (2012).

9. Uoyama, H., Goushi, K., Shizu, K., Nomura, H. \& Adachi, C. Highly efficient organic light-emitting diodes from delayed fluorescence. Nature 492, 234-238 (2012). 
10. Chen, P. et al. Delayed fluorescence in a solution-processable pure red molecular organic emitter based on dithienylbenzothiadiazole: a joint optical, electroluminescence, and magnetoelectroluminescence study. ACS Appl. Mater. Interfaces 7, 2972-2978 (2015).

11. Dias, F. B. et al. Triplet harvesting with $100 \%$ efficiency by way of thermally activated delayed fluorescence in charge transfer OLED emitters. Adv. Mater. 25 3707-3714 (2013).

12. Devi, L. S. et al. Triplet energy transfer in conjugated polymers. I. Experimental investigation of a weakly disordered compound. Phys. Rev. B 78, 045210 (2008).

13. Fishchuk, I. I. et al. Triplet energy transfer in conjugated polymers. II. A Polaron Theory Descr. Address. Influ. Disord. Phys. Rev. B 78, 045211 (2008).

14. Hoffmann, S. T. et al. Triplet energy transfer in conjugated polymers. III. Exp. Assess. Influ. Disord. Polaronic Transp. Phys. Rev. B 81, 165208 (2010).

15. Lu, L. P., Kabra, D. \& Friend, R. H. Barium hydroxide as an interlayer between zinc oxide and a luminescent conjugated polymer for light-emitting diodes. Adv. Funct. Mater. 22, 4165-4171 (2012).

16. Lee, B. R. et al. Highly efficient inverted polymer light-emitting diodes using surface modifications of ZnO layer. Nat. Commun. 5 https://doi.org/10.1038/ ncommS6840 (2014).

17. Stevens, M. A., Silva, C., Russell, D. M. \& Friend, R. H. Exciton dissociation mechanisms in the polymeric semiconductors poly(9,9-dioctylfluorene) and poly (9,9-dioctylfluorene-co-benzothiadiazole). Phys. Rev. B 63, 165213 (2001).

18. Gélinas, $S$. et al. Recombination dynamics of charge pairs in a push-pull polyfluorene-derivative. J. Phys. Chem. B 117, 4649-4653 (2012).

19. Donley, C. L. et al. Effects of packing structure on the optoelectronic and charge transport properties in poly (9, 9-di-n-octylfluorene-alt-benzothiadiazole). J. Am. Chem. Soc. 127, 12890-12899 (2005).

20. Cornil, J. et al. Electronic and optical properties of polyfluorene and fluorenebased copolymers: a quantum-chemical characterization. J. Chem. Phys. 118, 6615-6623 (2003).

21. Schmidtke, J. P., Kim, J.-S., Gierschner, J., Silva, C. \& Friend, R. H. Optical spectroscopy of a polyfluorene copolymer at high pressure: intra- and intermolecular interactions. Phys. Rev. Lett. 99, 167401 (2007).

22. Gerhard, A. \& Bässler, H. Delayed fluorescence of a poly (p-phenylenevinylene) derivative: triplet-triplet annihilation versus geminate pair recombination. J. Chem. Phys. 117, 7350-7356 (2002).

23. Rothe, C. \& Monkman, A. Regarding the origin of the delayed fluorescence of conjugated polymers. J. Chem. Phys. 123, 244904 (2005).

24. Hoffmann, S. T. et al. Spectral diffusion in poly (para-phenylene)-type polymers with different energetic disorder. Phys. Rev. B 81, 115103 (2010).

25. Lee, C.-L., Yang, X. \& Greenham, N. C. Determination of the triplet excited-state absorption cross section in a polyfluorene by energy transfer from a phosphorescent metal complex. Phys. Rev. B 76, 245201 (2007).

26. Rothe, C. \& Monkman, A. P. Triplet exciton migration in a conjugated polyfluorene. Phys. Rev. B 68, 075208 (2003).

27. Yan, M., Rothberg, L., Kwock, E. \& Miller, T. Interchain excitations in conjugated polymers. Phys. Rev. Lett. 75, 1992 (1995).

28. Irkhin, P. \& Biaggio, I. Direct imaging of anisotropic exciton diffusion and triplet diffusion length in rubrene single crystals. Phys. Rev. Lett. 107, 017402 (2011).

29. Hertel, D., Bässler, H., Guentner, R. \& Scherf, U. Triplet-triplet annihilation in a poly (fluorene)-derivative. J. Chem. Phys. 115, 10007-10013 (2001).
30. Donley, C. L. et al. Effects of packing structure on the optoelectronic and charge transport properties in poly(9,9-di-n-octylfluorene-alt-benzothiadiazole). J. Am. Chem. Soc. 127, 12890-12899 (2005).

31. Yang, G.-Z., Wang, W.-Z., Wang, M. \& Liu, T. Side-chain effect on the structura evolution and properties of poly(9,9-dihexylfluorene-alt-2,5-dialkoxybenzene) copolymers. J. Phys. Chem. B 111, 7747-7755 (2007).

32. Subash Sundar, T., Sen, R. \& Johari, P. Rationally designed donor-acceptor scheme based molecules for applications in opto-electronic devices. Phys. Chem. Chem. Phys. 18, 9133-9147 (2016).

33. Nikolaenko, A. E., Cass, M., Bourcet, F., Mohamad, D. \& Roberts, M. Thermally activated delayed fluorescence in polymers: a new route toward highly efficient solution processable OLEDs. Adv. Mater. 27, 7236-7240 (2015).

34. Ogiwara, T., Wakikawa, Y. \& Ikoma, T. Mechanism of intersystem crossing of thermally activated delayed fluorescence molecules. J. Phys. Chem. A 119, 3415-3418 (2015).

35. Chen, P. et al. Magneto-electroluminescence as a tool to discern the origin of delayed fluorescence: reverse intersystem crossing or triplet-triplet annihilation? Adv. Opt. Mater. 2, 142-148 (2014).

36. Gärditz, C., Mückl, A. G. \& Cölle, M. Influence of an external magnetic field on the singlet and triplet emissions of tris-(8-hydroxyquinoline) aluminum (III)(Al q 3). J. Appl. Phys. 98, 104507 (2005).

37. Johnson, R. \& Merrifield, R. Effects of magnetic fields on the mutual annihilation of triplet excitons in anthracene crystals. Phys. Rev. B 1, 896 (1970).

38. Kalinowski, J., Cocchi, M., Virgili, D., Di Marco, P. \& Fattori, V. Magnetic field effects on emission and current in Alq 3-based electroluminescent diodes. Chem. Phys. Lett. 380, 710-715 (2003).

39. Kohler, A. \& Bassler, H. What controls triplet exciton transfer in organic semiconductors? J. Mater. Chem. 21, 4003-4011 (2011).

40. Chen, T. et al. Understanding the control of singlet-triplet splitting for organic exciton manipulating: a combined theoretical and experimental approach. Sci. Rep. 5, 10923 (2015).

41. Zhou, J. et al. Charge-transfer-featured materials-promising hosts for fabrication of efficient OLEDs through triplet harvesting via triplet fusion. Chem. Commun. 50, 7586-7589 (2014)

Open Access This article is licensed under a Creative Commons Attribution 4.0 International License, which permits use, sharing, adaptation, distribution and reproduction in any medium or format, as long as you give appropriate credit to the original author(s) and the source, provide a link to the Creative Commons license, and indicate if changes were made. The images or other third party material in this article are included in the article's Creative Commons license, unless indicated otherwise in a credit line to the material. If material is not included in the article's Creative Commons license and your intended use is not permitted by statutory regulation or exceeds the permitted use, you will need to obtain permission directly from the copyright holder. To view a copy of this license, visit http://creativecommons. org/licenses/by/4.0/.

(c) The Author(s) 2018 